\title{
ESSAI DE CLASSIFICATION DES PHLÉBOTOMES
}

\author{
Par Virgil NITZULESCU
}

La classification des phlébotomes, proposée par França et Parrot. en 1921, ne correspond pas aux divisions naturelles du genre Phlebotomus. Des espèces très voisines se trouvent placées dans des sous-genres distincts, tandis que des formes beaucoup plus différentes entre elles par l'organisation se trouvent englobées dans un même sous-genre.

C'est pour remédier à cet inconvénient que Sinton, en 1927, propose une autre classification, dans laquelle il tient compte non seulement des caractères extérieurs, mais aussi de certains détails de la morphologie interne dont Adler et Theodor avaient montré la grande importance en taxonomie, tels que l'armature buccale et les spermathèques.

Le caractère extérieur auquel Sinton fait appel n'est plus l'armature génitale du mâle, qui avait servi à França et Parrot pour l'édification de leurs sous-genres, mais le caractère indiqué par Newstead dès 1911, résultant de la position des " poils " des tergites abdominaux, à l'exception du premier segment. Sinton divise en conséquence les phlébotomes en trois groupes: le premier groupe est celui des phlébotomes à poils abdominaux dressés, le second est celui à poils abdominaux couchés, et le troisième, représenté par la seule espèce $P$. squamipleuris, est un groupe intermédiaire. Cet auteur s'adresse à ce caractère des poils parce qu'il avait observé une relation entre leur disposition et/a forme de la spermathèque : dans le groupe à poils dressés la spermathèque est crénelée, dans celui à poils couchés elle est lisse et dans le groupe intermédiaire la spermathèque revêt une forme particulière.

Cette division, malgré son apparence séduisante, n'est pas satisfaisante, car le caractère de la disposition des poils ne possède pas toute la netteté désirable. A côté des espèces à poils dressés et à poils couchés, il en existe d'autres qui peuvent revêtir des formes intermédiaires ; c'est le cas de $P$. malabaricus, dont les poils sont, d'après l'observation de Sinton lui-même, disposés d'une manière " semi-recumbante ". A cause de sa spermathèque lisse, on a dû faire abstraction de la disposition des poils et englober le $P$. mala-

Annales de Parasitologie, T. IX, $\mathrm{n}^{\circ} 3 .-1^{\mathrm{er}}$ mai 1931, p. 271-275. 
baricus parmi les phlébotomes à poils couchés, dans le deuxième groupe.

D'autre part, le groupe à poils dressés est divisé par Sinton en deux sections. Dans la première section les poils dressés sont extrêmement nombreux, dans la seconde, en réalité, la majorité est représentée par des espèces à poils couchés et si la section se trouve réunie au groupe des phlébotomes à poils dressés, c'est à cause de la forme crénelée des spermathèques, forme qui doit, d'après Sinton, caractériser le groupe des phlébotomes à poils dressés. Pourtant, d'un côté, la corrélation entre la spermathèque crénelée et les poils abdominaux dressés n'est pas sans présenter des exceptions, car $P$. larroussei et $P$. chinensis possèdent des spermathèques lisses et des poils dressés; d'un autre côté, les phlébotomes de la deuxième section du premier groupe de Sinton possèdent une armature buccale. Or l'armature buccale est caractéristique du groupe des phlébotomes à poils couchés et s'accorderait mieux avec la disposition de la majorité de leurs poils.

On pourrait, en conséquence, mettre la deuxième division du premier groupe de Sinton tout aussi bien dans le premier groupe, comme le fait Sinton, que dans le deuxième groupe, si on tenait compte plutòt de l'armature buccale que de la spermathèque en même temps que de la disposition de la majorité des poils.

D'ailleurs rien ne plaide plus contre l'introduction du caractère de la position des poils dans la systématique des phlébotomes que l'existence d'un groupe intermédiaire dans lequel, par un simple hasard, des exemplaires de la même espèce peuvent revêtir un type ou un autre ( $\boldsymbol{P}$. squamipleuris).

Nous croyons done qu'il vaudrait mieux éliminer complètement le caractère de la disposition des poils abdominaux et ne retenir dans la classification que les caractères de la morphologie interne. C'est en nous basant sur ces caractères que nous proposons la division du genre Phlebotomus en deux sections, d'après l'existence ou l'absence d'une armature buccale. C'est un caractère net, facile à observer et tenant à des formations chitineuses. Il nous paraît des plus importants et les deux sections qu'il détermine correspondent à des groupes naturels.

Dans chacune de ces deux sections les phlébotomes femelles peuvent présenter des spermathèques à parois crénelées ou non crénelées. Il est nécessaire de préciser le sens du mot crénelé emprunté aux anciennes descriptions. Nous entendons par crénelé une segmentation purement externe, mais nette et profonde. La spermathèque du $P$. chinensis, parcourue par des lignes qui se croisent à 
divers angles, est non crénelée, malgré l'apparence ficelée qu'elle prend dans certaines préparations des exemplaires de Chine, d'après les dessins de Sinton. De même nous considérons comme non crénelée la spermathèque du $P$. taianensis. La spermathèque du $P$. squamipleuris doit être rangée aussi parmi les spermathèques non crénelées.

Nous aurons, de cette manière, divisé tous les phlébotomes en 4 groupes :

Armature buccale présente $\left\{\begin{array}{l}\text { Spermathèque crénelée........... } 1 \\ \text { Spermathèque non crénelée }\end{array}\right.$

Armature buccale absente $\{$ Spermathèque crénelée......... 3

Spermathèque non crénelée...... 4

1. Le premier groupe de cette classification renferme en entier le groupe de Sinton à poils en majorité couchés et à spermathèques crénelées, dont cet auteur faisait la deuxième division du groupe à poils dressés. Nous croyons qu'on pourrait avantageusement transformer ce groupe si bien caractérisé en un sous-genre que nous sommes heureux d'appeler Sintonius, en hommage à Sinton qui l'a particulièrement étudié. Dans la faune asiatique, nous citons comme Sintonius, $P$. hospitii, P. christophersi, $P$. clydei. Dans la faune européenne, ce sous-genre n'est pas représenté. Dans la faune américaine, nous avons déjà donné la description de la femelle du $P$. intermedius du Brésil et du $P$. gomezi du Venezuela.

2. Le deuxième groupe comprendra les phlébotomes à armature buccale et dont la spermathèque n'est pas crénelée. Il correspondra au deuxième groupe de Sinton (divisions 1 et 2). Les limites entre les deux divisions de Sinton ne sont pas suffisamment nettes. Elles ne paraissent pas correspondre à des groupes naturels et nous les réunissons dans le même sous-genre dont le type est $P$. minutus et qui renferme toutes les autres espèces qu'on réunissait auparavant sous le nom de groupe minutus. Nous substituons à cette expression, dont personne n'a jusqu’à présent précisé le sens, le sousgenre Brumptius (1), et nous y faisons entrer tous les phlébotomes présentant les caractères de notre deuxième groupe.

3. Dans notre troisième groupe, nous plaçons les formes privées d'armature buccale et dont la femelle possède des spermathèques crénelées. Ces formes peuvent être représentées par deux types : $P$. major et $P$. papatasi.

(1) Nous dédions avec reconnaissance ce sous-genre au Prof. Brumpt qui a bien voulu nous accueillir à plusieurs reprises pour de longs séjours dans son laboratoire et nous communiquer ses riches collections de phlébotomes.

AnNales de Parasitologie, T. IX, $\mathrm{n}^{\circ} 3 .-1^{\text {er }}$ mai 1931. 
Autour du $P$. major se groupent une certaine quantité d'espèces qui lui ressemblent plus ou moins. Ce sont : $P$. perniciosus et sa variété tobbi, $P$. ariasi, $P$. langeroni et sa variété longicuspis, $P$. kandelaki, $P$. wenyoni. Toutes ces espèces présentent les caractères communs suivants : le pharynx est armé de dents fines et petites, disposées comme des épines sur des fractions de lignes entremêlées. La spermathèque des femelles est crénelée et pourvue d'un long col.

Ces caractères avaient été considérés, au moment de leur découverte, comme propres à $P$. major et les phlébotomes qui les présentaient étaient englobés dans cette espèce. C'est ainsi que Sinton, après examen de l'armature buccale et pharyngienne du $P$. perniciosus, fait de cette espèce une simple variété du $P$. major. Cette manière de voir n'est pas justifiée. Nous avons déjà montré, en 1930, que les limites déterminées par les caractères du pharynx et de la spermathèque sont trop larges et que l'on doit rechercher, au sein de ces démarcations, des caractères secondaires qui délimiteront les espèces.

C'est la forme du pénis du mâle qui donnera des caractères spécifiques, cet organe présentant, dans chaque espèce du groupe major, une forme différente et caractéristique. $P$. wenyoni seul fait exception, car il est caractérisé par la multiplicité des dents pharyngiennes et non par la forme du pénis, mais $P$. wenyoni ne devrait pas, à notre avis, être considéré comme une espèce indépendante. Nous le ramenons au rang de variété du $P$. major stricto sensu.

Jusqu'ici les mots " stricto sensu " devaient nécessairement être ajoutés au nom du $P$. major pour éviter la confusion entre cette espèce et ce que l'on appelle le "groupe major », c'est-à-dire la totalité des phlébotomes dont nous venons d'indiquer les caractères. Le groupe major est important à conserver, car les espèces qui le forment sont nettement apparentées, mais nous le faisons entrer dans notre classification en le transformant en sous-genre que nous nommons Larroussius (1) et dont le type est $P$. major.

Pour les autres phlébotomes, compris dans notre troisième groupe et qui ne sont pas des Larroussius, nous proposons le sousgenre Phlebotomus conformément à l'article 9 du Code de nomenclature zoologique. Les femelles possèdent des spermathèques crénelées, à cou très court. Le type du sous-genre Phlebotomus est l'espèce la plus anciennement décrite, $P$. papatasi. Le $P$. sergenti rentre aussi dans ce sous-genre.

(1) Nous sommes heureux de dédier ce sous-genre au Dr Larrousse dont la monographie (1921) constitue la première étude d'ensemble consacrée aux phlébotomes. 
Il nous reste à compléter notre classification par le cinquième groupe, c'est-à-dire par les phlébotomes dépourvus d'armature buccale et dont les femelles présentent des spermathèques lisses. Nous connaissons deux espèces qui doivent y entrer : $P$. chinensis et $P$. larroussei. Nous les groupons dans un sous-genre à part que nous nommons Adĩerius, en le dédiant à Adler dont les découvertes ont fait faire tant de progrès à l'étude des phlébotomes et nous servent de point de départ pour cet essai de classification.

\section{RÉsumé}

Nous divisons le genre Phlebotomus en 5 sous-genres, à chacun desquels nous rattachons les espèces dont les caractères nous sont connus avec certitude.

\section{A. Phlébotomes a armature buccale.}

I. Spermathèques crénelées :

Sintonius. Type : $P$. hospitii. Principales espèces : $P$. clydei, $P$. christophersi, P. intermedius, P. gomezi, P. philippinensis.

II. Spermathèques non crénelées :

Brumptius. Type : $P$. minutus. Principales espèces : $P$. africanus, $P$. barraudi, $P$. brodeni, $P$. collarti, $P$. demeijerei, $P$. heizeri, $P$. hitcheni, $P$. ingrami, $P$. manganus, $P$. nicnic, $P$. palestinensis, $P$. parroti, $P$. sanneri, $P$. schoutedeni, $P$. schwetzi, $P$. shorti, $P$. simillimus, P. squamipleuris, P. squamirostris.

B. Phlébotomes sans armature buccale.

III. Spermathèques crénelées à col plus ou moins long, pharynx comme chez $P$. major :

Larroussius. Type : $P$. major. Principales espèces : $P$. perniciosus, $P$. ariasi, $P$. langeroni, $P$. kandelaki.

IV. Spermathèques crénelées à col court :

Phlebotomus. Type: P. papatasi. Principales espèces: P. sergenti, $P$. caucasicus, $P$. newsteadi, $P$. argentipes.

V. Spermathèques lisses :

Adlerius. Type : $P$. chinensis. Autre espèce : P. larroussei.

Laboratoire de Parasitologie de la Faculté de Médecine de Paris. 